\title{
THEORY OF PHOTON AND ELECTRON INDUCED REACTIONS
}

\author{
DOE/ER/40370--5 \\ Final Report for Period \\ DE93 005925 \\ July 1, 1990 - June 30, 1993
}

David S. Onley and Louis E. Wright

Institute of Nuclear and Particle Physics

Deparrment of Physics and Astronomy

Ohio Lniversity

Athens. Ohio 45701

January 1993

\section{DISCLAIMER}

This report was prepared as an account of work sponsored by an agency of the United States Government. Neither the United States Government nor any agency thereof, not any of their employees, makes any warranty, express or implied, or assumes any legal liability or responsibility for the accuracy, completeness, or usefulness of any information, apparatus, product, or process disclosed, or represents that its use would not infringe privately owned rights. Reference herein to any specific commercial product, process, or service by trade name, trademark, manufacturer, or otherwise does not necessarily constitute or imply its endorsement, recommendation, or favoring by the Unitad States Government or any agency thereof. The views and opinions of authors expressed herein do not necessarily state or reflect those of the United States Government or any agency thereof.

The Cnited State Department of Energy

Grant o. DE-FG02-8iER 40370 


\section{PROGRESS REPORT, JANUARY 1993}

During the first two and one half years of the current grant from the Department of Energy we have been quite successtul and have made significant progress on the following aspects of the general investigation of electron and photon induced reactions: (a) photo- and electro-production of mesons; (b) Coulomb distortion effects on $\left(e, e^{\prime} \gamma\right)$ and on inclusive $\left(e, e^{\prime}\right)$ and exclusive $\left(e, e^{\prime} p\right)$ scattering in the quasi-elastic region; (c) relativistic structure calculations, and (d) quark models. We will report on each of these developments in the following sections:

\section{(a) Photo- and Electro-Production of Mesons}

Early in the grant period, our investigation of lambda polarization in the reaction $\gamma+d \rightarrow K^{+}+n+\Lambda^{0}$ was completed [1]. We looked at three different lambda-neutron potentials which have been proposed by others, but which have quite different scattering lengths and effective ranges. We found that under certain reasonable kinematical conditions the exclusive reaction cross section and the polarization vary enough with the choice of potential to be useful in determining the lambda-neutron interaction. As we pointed out earlier, this reaction is being actively proposed for the Large Acceptance Angle Spectrometer being planned at CEBAF [2], and we await experimental results.

In collaboration with $\mathrm{C}$. Bennhold at TRIUMF (now at George Washington University), we also completed the extension of this calculation to study kaon photoproduction from the neutron by using the deuteron as a target. We used basic kaon photoproduction operators proposed by Bennhold [3], although there exist no experimental data to test them due to lack of a neutron target. We found that it is feasible to use the deuteron as a neutron target over a large kinematic region without the final state interaction of the outgoing meson and spectator nucleon causing any problems. Our paper reporting our findings has appeared [4]. All six kaon photoproduction reactions from the deuteron that we investigated are scheduled for the Large Aiceptance Angle Spectrometer being built at CEBAF [2]. In this regard, we also worked out a convenient method of making a multipole decomposition of kaon photoproduction amplitudes based on Feynman techniques i.j.].

During the past year we have been investigating the exclusive reaction $A(\gamma, \pi N) B$ which offers a mechanism for studying medium effects on nucleons, the delta and the nucleon delta transition. While the impulse approximation has been very successful in describing $(\gamma, \pi)$ reactions on p-shell nuclei [6] below the delta region, when the photon energy reaches about $300 \mathrm{MeV}$ the agreement with $(\gamma, \pi)$ experiments deteriorates[ $[\bar{l}$. Note that at resonance. the delta contribution for charged pions is approximately equal to the background contribution arising from the Born terms. In $(\gamma, \pi)$ the final nucleon is still bound and thus nuclear structure plays a significant role which may obscure possible modifications of the delta by the medium. However, by detecting the pion and the nucleon in coincidence in the continuum. one may get a handle on 
the properties of the $\Delta$ in the nuclear medium. We look at the quasifree region where the momentum transfer to the residual nucleus is relatively low under $300 \mathrm{MleV} / \mathrm{c}$. which will remove any strong dependence on the details of the single-particle nuclear wavefunctions.

Data for the exclusive reaction on complex nuclei in the $\Delta$ region are sparse. There is a recent review article by Steenhoven [8] which summarizes the experimental situation to date. A. number of experiments on ${ }^{12} C$ have been done at the Tomsk synchrotron $[9,10]$, and an experiment on ${ }^{16} O$ has been done at MIT/Bates [11]. Such coincidence experiments are difficult to perform without a high duty cycle machine with the capability of having a well defined photon beam energy and good energy resolution for particle detection. In the case of the Bates experiment in the $\Delta$ region. the statistics are poor and error bars are large, and the coincidence cross section was integrated over the pion energy thus making it not truly exclusive. The Tomsk experiment suffers from a not-well defined photon beam. With the advent of improved or new high duty cycle accelerators such as LEGS at Brookhaven. CEBAF. Bates. NIKHEF and MAMI, and with better particle detection techniques. more accurate data should become available in the near future. This will greatly enhance our knowledge of many aspects of pion photoproduction from complex nuclei. In fact, there has recently been an approved proposal by Hicks [12] to measure cross sections and photon asymmetries on ${ }^{12} \mathrm{C}$ using the LEGS facility at Brookhaven. The preparation work on the experiment is ongoing. Also. NIKHEF is planning to do the exclusive experiments on ${ }^{12} \mathrm{C}$ and ${ }^{4} \mathrm{He}[8]$.

Fig. 1 is a diagramatic illustration of DWI.A: a photon penetrates the nucleus. interacts with a single bound nucleon and excites a delta: the delta propagates inside the nucleus resulting in the emission of a pion and a nucleon. the outgoing pion and nucleon interact with the remaining nucleons while leaving the nucleus. Of course. the non-resonant (Born) terms also contribute coherently:

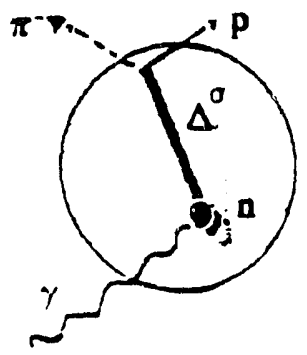

Fig. 1. DIVIA for the exclusive reaction $i(\because, \pi) B$.

Formalism- Ve use harmonic oscillator wavetunctions to describe the bound nucleon. More realistic Woods-Saxon wave functions can be incorporated if necessary. For the pion production process. we use the full Blomqvist-Laget production operator [13.11] along with the second $\gamma N \Delta$ gauge coupling in the $\lambda$ channel [15] without the various approximations that are commonly used in earlier studies. We have set up our calculation to handle both charged and uncharged pions. Here we just look at photoproduction of $\left(\pi^{-} p\right)$. For the spectroscopic factors. we use ralues determined by (e.e'p) experiments. For the pion optical potential. we use the one developed by Stricker. Mc.Manus and Carr [16]. For the nucleon optical potential. we use a parametrization provided by Schwandt [1i]. 
Following standard procedures, the coincidence differential cross section for $A(\gamma, \pi \mathrm{N}) \mathrm{B}$ in the lab trame. where the the incident photon defines the z-axis, and the outgoing pion lies in the $x-z$ plane. can be written as

$$
\frac{d^{3} \sigma}{d E_{\pi} d \Omega_{\tau} d \Omega_{N}}=\frac{. M_{f} m_{N} q p}{4(2 \pi)^{5} E_{\gamma}\left|E_{N}+E_{f}-E_{N v} \mathrm{p} \cdot(\mathbf{k}-\mathbf{q}) / p^{2}\right|} \sum\left|\cdot M_{f i}\right|^{2}
$$

where $\bar{\Sigma}$ means sum over final spins and average over initial spins if no polarization is observed. After performing the sums, we obtain

$$
\bar{\sum}\left|M_{f i}\right|^{2}=\frac{1}{2\left(2 J_{i}+1\right)} \sum_{j m \lambda m,} \frac{S_{j}}{2 j+1}\left|T\left(j, m, \lambda, m_{g}\right)\right|^{2},
$$

where $(\mathrm{jm})$ is the quantum number of the bound nucleon, $\lambda$ is the photon polarization and $m$, is the spin projection of the outgoing nucleon. $S_{j}$ is the spectroscopic factor. The matrix element $\mathrm{T}$ is given by

$$
T\left(j, m . \lambda, m_{\mathbf{s}}\right)=\int d^{3} p^{\prime} d^{3} q^{\prime} \Psi_{m_{s}}^{(+)}\left(\mathbf{p}^{\prime}, \mathbf{p}\right) \phi_{\pi}^{(+)}\left(\mathbf{q}^{\prime}, \mathbf{q}\right) t_{\gamma \pi}\left(\lambda, \mathbf{k}, \mathbf{p}_{i}, \mathbf{q}^{\prime}, \mathbf{p}^{\prime}\right) \Psi_{j m}\left(\mathbf{p}_{i}\right)
$$

where $\mathrm{p}_{i}=\mathrm{p}^{\prime}+\mathrm{q}^{\prime}+\mathrm{k}$ is the momentum of the bound nucleon.

The pion production operator $t_{\gamma \pi}$ depends strongly on various momenta. and thus would be a nonlocal operator in coordinate space. Working in momentum space allows a straightforward treatment of all nonlocal effects arising from the production operator without approximation. However. evaluation of the 6-dimensional integral in Eq. (3) must be done numerically and coupled with the needed summations can only be accomplished with the help of a supercomputer such as a Cray. Our code takes advantage of the vectorization and optimization capabilities of the Cray Y'-MP at the Ohio Supercomputer Center.

It is useful to discuss some approximations that are commonly made. Firstly, in Eq. (3). if we fix the pion and proton momenta at their asymptotic values. i.e., we make the production operator $t_{\alpha_{\pi}}$ local. then the matrix element can be easily carried out (local DIIIA approximation). This approximation clearly reduces the computational time needed and we have investigated its validity. Further. if we replace the pion and nucleon wavefunctions with plane waves. the matrix element takes the simpli: form of the production operator multiplied by the Fourier transform of the single particle bound wavefunction (FIVIA approximation).

Comparisons with Data-Fig. 2 shows the comparison between one experiment done at the Tomsk synchrotron [9] and the PIVIA. local DWVIA and nonlocal DWVIA calculations for the case of $\left(\pi^{-} p\right)$ production from the neutrons in the $1 s_{1 / 2}$ and $1 p_{3 / 2}$ shells of ${ }^{12} \mathrm{C}$. The triple coincidence cross section are plotted as a function of the proton energy for fixed photon energy and backward $\left(120^{\circ}\right)$ pion angle and forward proton angle $\left(20^{\circ}\right)$. The pions and protons were detected in coincidence in the production plane by two spectrometers sitting on opposite sides of the photon beam. Lnder these 


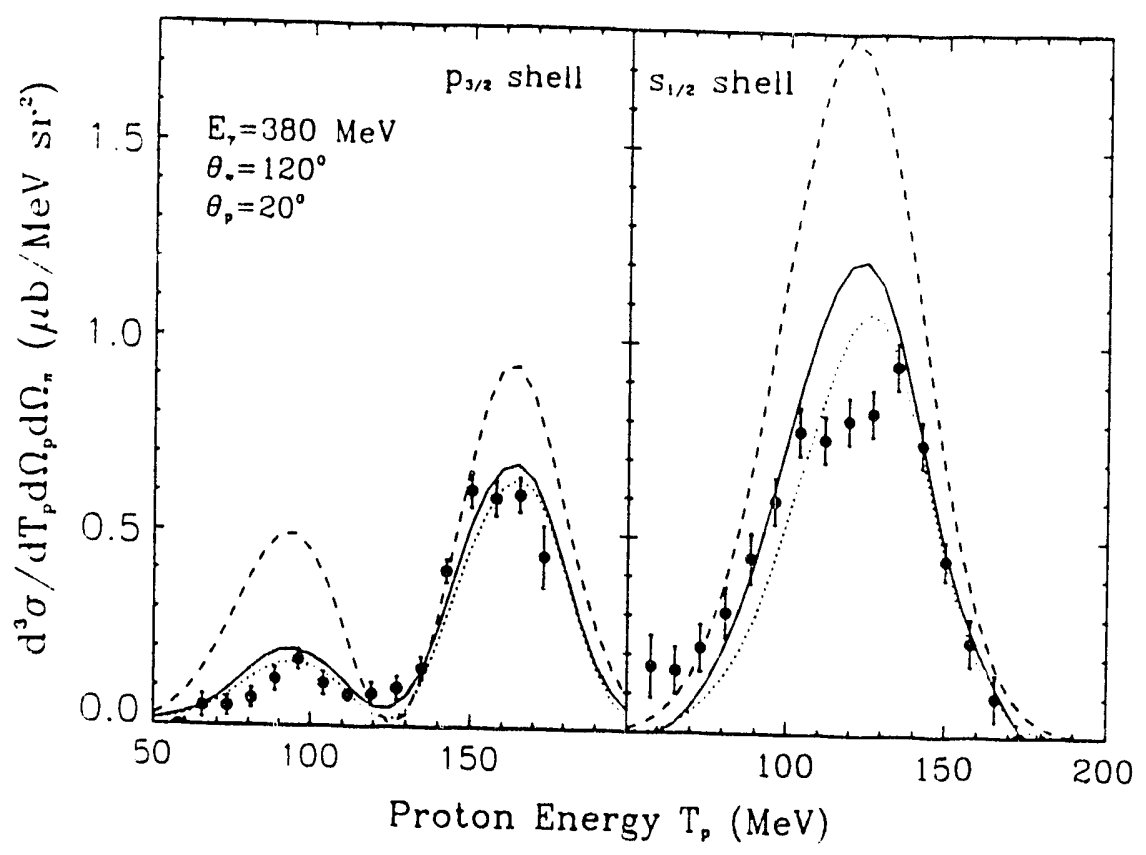

Fig. 2 Proton energy dependence of the triple coincidence cross section from $p_{3 / 2}$ and $s_{1 / 2}$ shell
neutrons in 12 (dashed line). local DVIIA for fixed $E_{\gamma}, \theta_{\pi}$ and $\theta_{p}$. Theoretical curves are calculated in PWIA Ref. [9]

kinematics. the pion energy $T_{\pi}$, the momentum transfer $\mathrm{Q}$ and the invariant mass $W$ all change with $T_{p}$.

For example. in the case of the 1s shell, in the range of $T_{p}$ from 50 to $190 \mathrm{MeV}$.

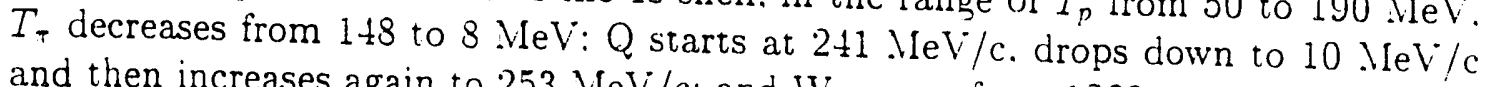
and then increases again to $253 \mathrm{MeV} / \mathrm{c}$ : and $\mathrm{W}$ ranges from 1260 to $1130 \mathrm{MeV}$. The kinematics for the $1 \mathrm{p}$ shell are similar. only differing because of the different binding energy. The shapes of the curves follow the momentum distribution of the bound neutron. namely, the minimum in $Q$ corresponds to the minimum of the cross section in the $1 \mathrm{p}$ shell case and the maximum in the $1 \mathrm{~s}$ shell case. Thus these kinematics can be used to obtain information on the neutron wavefunctions. which was one of the purposes of the experiment. The DWIA calculations give a good description of the cross section measured in terms of both shape and magnitude. while the PIVIA overestimates the data considerably. The distortion effects from final state interactions reduce the cross section and are important in explaining the data. Nonlocai DIVIA tends to enhance the local DWIA results. but the enhancement is not very large. only a few percent in both cases. Including nonlocal effects slightly improves the agreement between the theory and the experiment. Note that we have used s-shell and p-shell spectroscopic factors of $S_{s_{1 / 2}}=1.3$ and $S_{p_{3 / 2}}=2.6$. In Ref. [9]. a factorized DWIA with eikonal approximation was used to analyze the data. and it also gives a reasonable description of the data. However. we could not tell whether or not a 


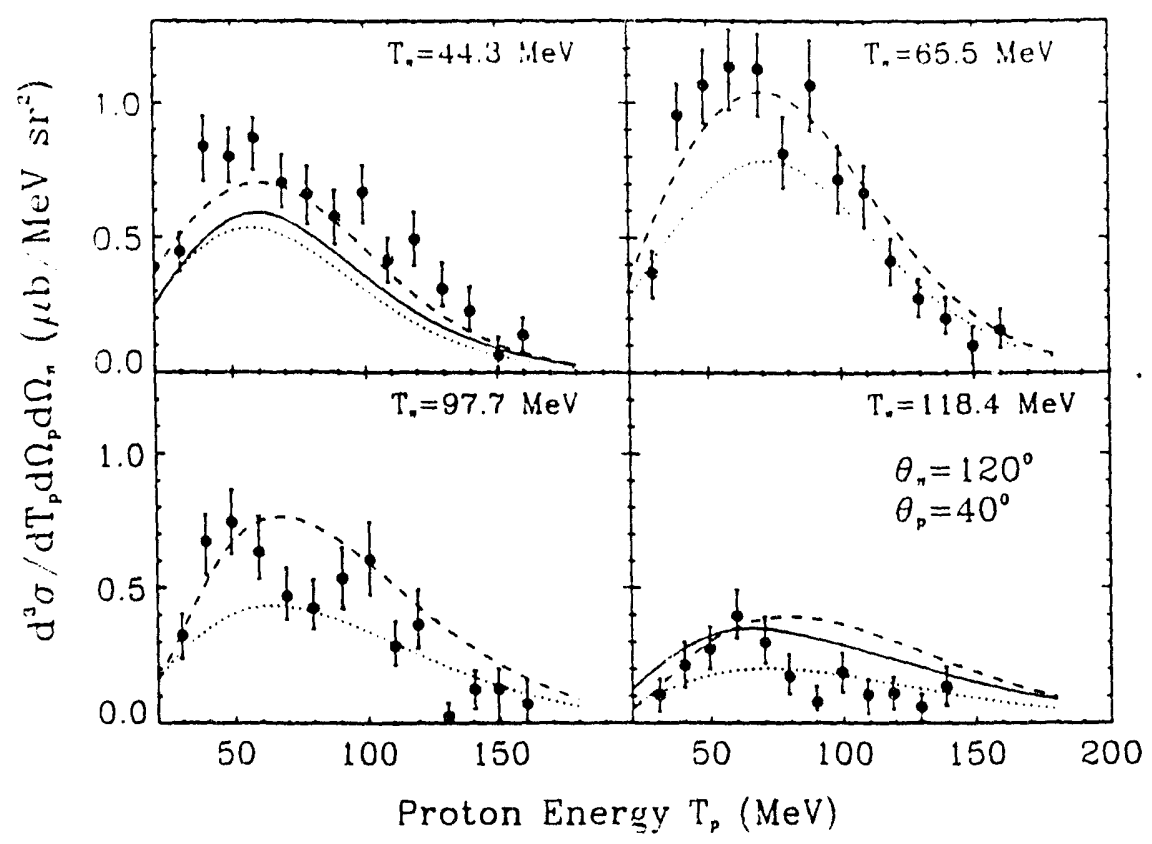

Fig. 3 Proton energy distributions of the differential cross section from combined $s$ and $p$ shell neutrons in ${ }^{12} \mathrm{C}$ at fixed $\theta_{\pi}$ and $\theta_{p}$ are shown at 4 different pion energies. Theoretical curves are calculated in PWIA (dashed line), local DWIA (dotted line) and nonlocal DWIA (solid line). Data are taken from Ref. [10].

spectroscopic factor was used in their analysis.

Fig. 2 shows the comparison with another experiment on ${ }^{12} C\left(\gamma, \pi^{-} p\right)^{11} C$ from Tomsk [10]. The combined s- and p-shell contributions to the triple cross section are shown as a function of the proton energy for four different pion energies 44.3. 65.5. 97.7 and $118.4 \mathrm{MeV}$. The pion angle is held at $120^{\circ}$ and proton angle at $40^{\circ}$ on the other side of the photon beam. In this kinematic setup. the photon energy $E_{\gamma}$ the invariant mass $W^{\circ}$ and the momentum transfer $\mathrm{Q}$ are not fixed. Examining the $1 \mathrm{p}$ shell separately. both $E_{\sim}$ and $\mathrm{W}$ increase with $T_{p}$, while $\mathrm{Q}$ has minima located at $T_{p}$ approximately equal to 40.50 .75 and $90 \mathrm{MeV}$ for each of the four pion energies. The values of these $Q_{\min }$ are 116.130 .155 and $173 \mathrm{MeV} / \mathrm{c}$. respectively. The data are somewhat higher than the theoretical curves while the shapes are reasonably reproduced. Again including nonlocal effects improves the agreement between our calculation and the data. The same spectroscopic factors as in the previous experiment are used. More detailed comparisons are not permittec' because of the relatively large error bars and the scatter in the data. especially in the $T_{T}=97.7 \mathrm{MeV}$ and $T_{\pi}=118.4 \mathrm{MeV}$ cases. The photon energy in this experiment was not very well

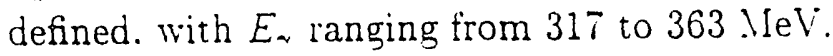

In Fig. t the PIVIA and local DWVIA calculations are compared to the experimental data from Bates [11] for the reaction ${ }^{i 6} O\left(\gamma, \pi^{-} p\right)^{15} O$. The experimental setup allows out of plane measurements. Data were taken at two pion angles. $64^{\circ}$ and $120^{\circ}$. 


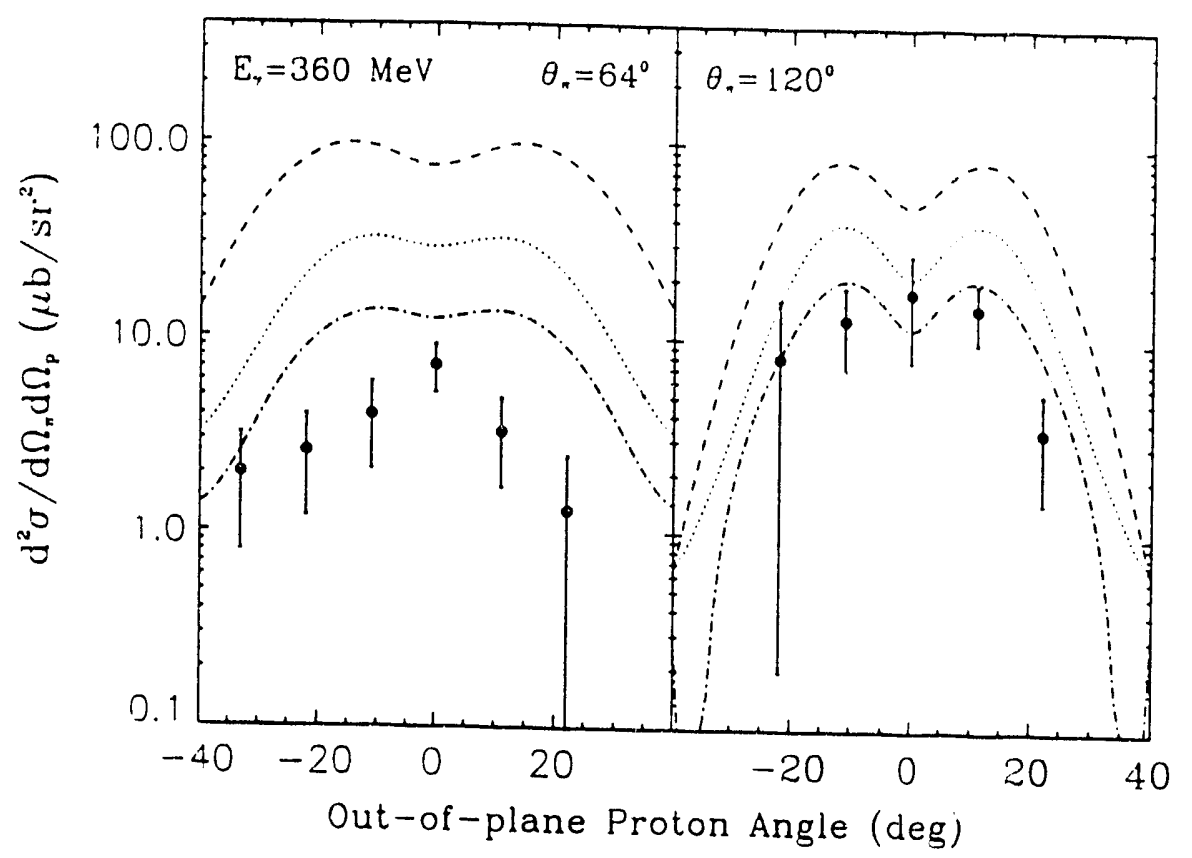

Fig. $\neq$ Out-of-plane proton angular dependence of the integrated coincidence cross section from combined $p_{3 / 2}$ and $p_{1 / 2}$ shell neutrons in ${ }^{16} O\left(\gamma, \pi^{-} p\right)^{15} \mathrm{O}$ for fixed photon energy and two pion angles. Theoretical curves are calculated in PWIA (dashed line), local DWIA (dotted line). The dashed-dotted curve is calculated in local DWIA with the $\Delta$ mass reduced by $j \%$. Data are taken from kief. [11].

while an vertical array of proton detectors were positioned at proton angles $40^{\circ}$ and $20^{\circ}$ corresponding to free two-body kinematics with the momentum transfer being zero. The out-of-plane proton angle goes from $33^{\circ}$ below the scattering plane to $33^{\circ}$ above the scattering plane corresponding to the momentum transfer $\mathrm{Q}$ from about $190 \mathrm{MeV} / \mathrm{c}$ to 0 then to $100 / \mathrm{c}$ again. The forward pion case corresponds to higher pion energy $(145 \mathrm{MeV})$ and lower proton energy ( $58 \mathrm{MeV})$, while the backward pion setup is just the opposite: lower pion energy ( $89 \mathrm{MeV})$. higher proton energy $(114$ Mel). The invariant mass does not change much. from 1225 to $1223 \mathrm{MeV}$. which stays in the $\rightarrow$ region.

Unfortunately, due to low counting rates. the data were integrated over a wide range of pion energies and therefore are not truly exclusive. This introduces a great deal of uncertainty in the data because each data point now contains a wide spectrum of momentum transfer Q. In making our comparisons we have tried to carry out the same integrations as done experimentally. Our calculations retain a slight minimum at $Q=0$ (in the scattering plane) while the experimental data do not. As expected. distortion reduces the cross section by about a factor of 2 to 4 . but does little to change the shape and location of the peaks. Our calculations include contributions from the $p_{3 / 2}$ and $p_{1 / 2}$ shells and we use a combined spectroscopic factor of $S_{1 p}=3.6$. The local DIVIA calculations are in reasonable agreement with the data for backward 
pions, but exceed the data for forward pions by about a factor of 3 . A factorized local DWVIA calculation used in Ref. [11] gives a similar description of the data. Clearly there is a problem at the forward pion angle. One difference between the forward and backward pion angles is that the forward pion angle corresponds to higher pion energy. Another differ nce is that the $\Delta$ contribution as compared to the Born contribution is larger at the forward pion angle. The problem may be that the pion optical potential used is not adequate, but it does successfully describe the Tomsk data for the same pion energies. This suggests that the production process may be modified by the nuclear medium.

We want to point out here that the kinematics for the Tomsk experiments and the Bates experiment are not the same. In the Tomsk experiments, energy dependence of the reaction is studied for the backward pion angle $\left(120^{\circ}\right)$ only, while in the Bates experiment angular dependence of the reaction is investigated for both forward and backward pion angles. Thus the two data sets do not check each other. The discrepancy between theory and data for forward pions stands on its own and deserves attention from both theory and experiment.

Suggestions for Future Experiments-In order to concentrate on the basic process. we need to keep the effects of final state interaction constant and the magnitude of the momentum transfer to the recoiling nucleus constant. This can be accomplished by the following simple kinematic conditions where we restrict ourselves to coplanar geometry for simplicity. We specify $E_{\gamma}, Q, T_{\pi}, \theta_{\pi}$ and $\phi_{N}=180^{\circ}$ in the energymomentum conservation equations. and solve for $T_{N}, \theta_{N}, \theta_{Q}$ and $\phi_{Q}$. In this way, the lengths of all external momenta ( $k, p, q$ and $Q$ ) are fixed, although the pion ind nucleon angles can be varied to obtain an angular distribution which depends sensitively on the basic production operator. Of course the distortions from pion and nucleon optical potentials still influence the cross section. but they are evaluated at the same energy over the complete angular range.

In Fig. $j$ we show the pion angular dependence of the cross section and the photon asymmetry calculated in PIVIA. local DWIA and the full nonlocal DWVIA for photon energy $E_{\sim}=360 \mathrm{MeV}$. momentum transfer $\mathrm{Q}=150 \mathrm{MeV} / \mathrm{c}$ and pion energy $T_{\pi}=100 \mathrm{MeV}$. With these values. the pion angle can only range from $60^{\circ}$ to $180^{\circ}$. More forward pion angles can be explored by increasing $Q$ and $T_{T}$ or decreasing $E_{\sim}$. However, we should point out that higher $Q$ will cause the counting rate to decrease: higher $T_{\pi}$ will result in more uncertainties in pion distorted waves: lower $E_{\gamma}$ will decrease the effects we are interested because we are going away from the $\checkmark$ region. The proton energy for these kinematics is $E_{p}=103 \mathrm{MeV}$ and the proton angle $\theta_{-}$ranges from about $41^{\circ}$ to $13^{0}$. The invariant mass $\mathrm{V}$ goes from 1197 to $1253 \mathrm{MeV}$. Conlocal effects enhance the local DVIA cross sections. The interesting feature is that the photon asymmetry is insensitive both to distortions and to nonlocal effects. This removes the uncertainties that may obscure the information from the production prusess. In this sense it is a relatively 'clean' observable and therefore should be considered in planning future experiments. 


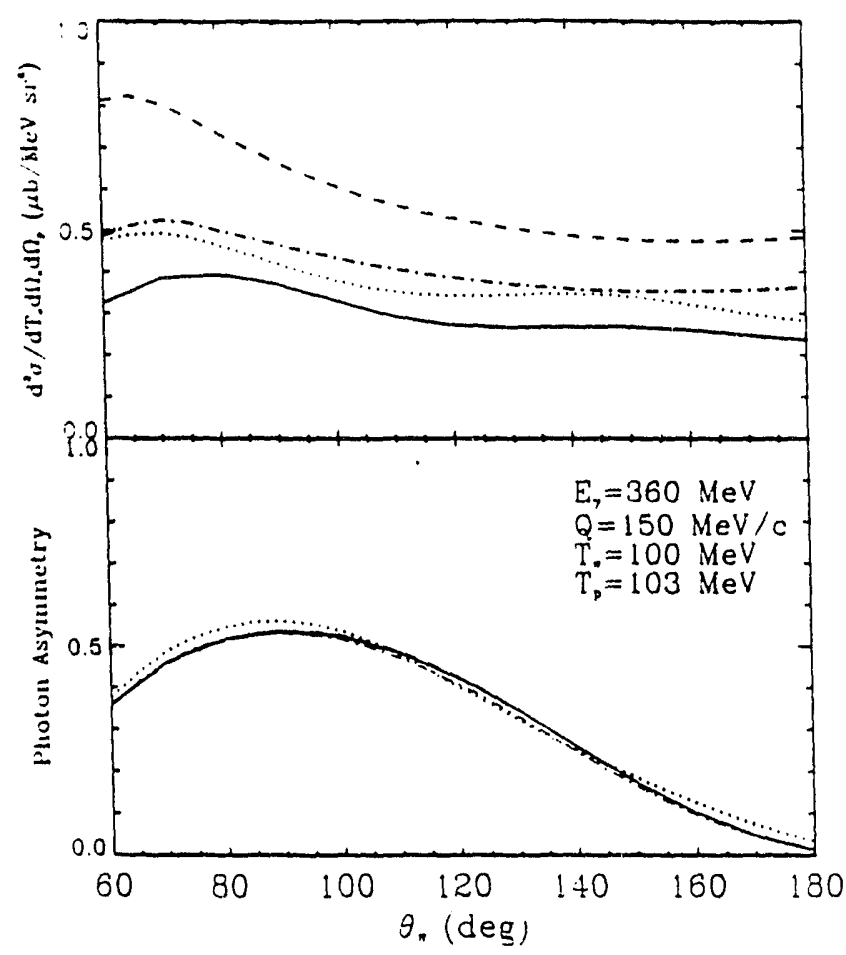

Fig. 5 The cross section and the photon asymmetry are shown in the 'fixed' kinematics specified in the text for $p_{3 / 2}$ neutrons in ${ }^{12} C\left(\gamma, \pi^{-} p\right)^{11} C$. Curves are calculated in PWIA (dashed line). local DWIA (solid line) and nonlocal DWIA (dotted line for pion distortion only, dot-dash line for proton distortion only).

We are interested in possible medium modifications of the $\Delta$. In the model of Blomqvist and Laget $[13,14]$, the mass $M_{\Delta}$, width $\Gamma_{\Delta}$ and coupling constant $G_{3}$ of the $\Delta$ were treated as free parameters and fitted to the elementary pion photoproduction data. In the nuclear medium. these parameters are all subject to modifications, thus they could be treated as 'effective' parameters. The sensitivity to various parameters in the calculation can help direct future experiments. In Figs. 6 and $T$ we show the effects of possible medium modifications by increasing and decreasing $M_{\perp}, \Gamma_{\perp}, G_{3}$ and as a demonstration the nucleon mass $m_{N}$ by $3 \%$. Obviously there is a great sensitivity to small variations in the $\Delta$ mass both in the cross section and in the photon asymmetry compared to very little sensitivity to the other parameters. In the language of the $\Delta$-hole theory, it is mainly the spreading potential resulting from the \lrcorner -nucleus interaction that gives this effective mass.

As a matter of fact. the discrepancy discussed in the Bates experiment is largely removed by reducing the $\Delta$ mass by about $5 \%$ in our local DIVIA calculation (see the dashed-dotted curve in Fig. 1). Moreover. the reduction in the calculation is differential for forward and backward pion angles. namely. it reduces the cross section more at the forward pion angle than at the backward pion angle. The same differential effects are also displaved here in Figs. 6 and $\tau$. This reduction of the $\triangle$ mass is clearly speculative and more dynamic studies coupled with better data are needed before any. conclusion can be reached. 


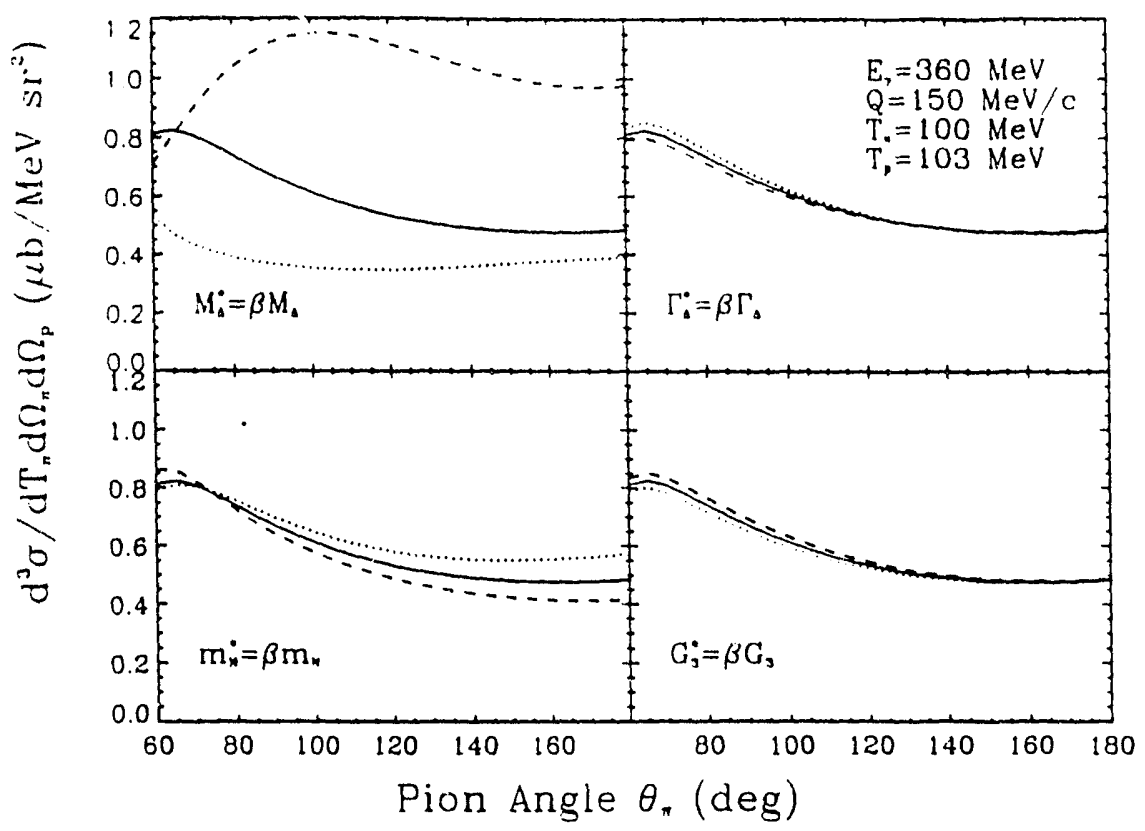

Fig. 6 Effec $:$ if possible medium modifications on the cross sections. Here $M_{\Delta}, \Gamma_{\Delta}$ and $G_{3}$ are the mass, width ard coupling constant of the $\Delta$. respectively, and $m_{N}$ is the nucleon mass. The dashed line is for $3=1.03$, dotted line for $\beta=0.97$ and solid line for $\beta=1.00$. The calculation is done in PIVIA.

Conciusions-From the discussions above we come to the following conclusions. The exclusive $A(\gamma, \pi \nu) E$ reaction offers an ideal laboratory for studying the $\Delta$ resonance in the nuclear medium. It allows for more direct access to the $\Delta$ than the reaction $A(\gamma, \pi) B$ since the final nucleon is no longer bound and the sensitivity to the nuclear structure of the target is thereby greatly reduced. The only information required for the target is the single particle bound wavefunction and the spectroscopic factor. which is only an overall factor in the cross section.

Kinematically, the reaction provides a great deal of flexibility since the target can take up a wide range of momentum transfer and for finite nuclei. little energy. We have proposed a kinematic arrangement that can best expose the information from the production vertex by fixing the lengths of each momentum vector in the overall momentum conservation.

The photon asymmetry is a very good observable to complement the cross section measurements. It comes mainly from the $\triangle$ resonance. is free from normalization problems. is predicted to be large and is relatively insensitive to the distortions. It should definitely be pursued at accelerators with the capability of polarized photon beams.

As far as the local DWVIA calculation is concerned, the distortions from final state interactions always reduce the PVIA results. Although distortion plays an important role in getting the correct magnitudes. it does little to change the shape of 


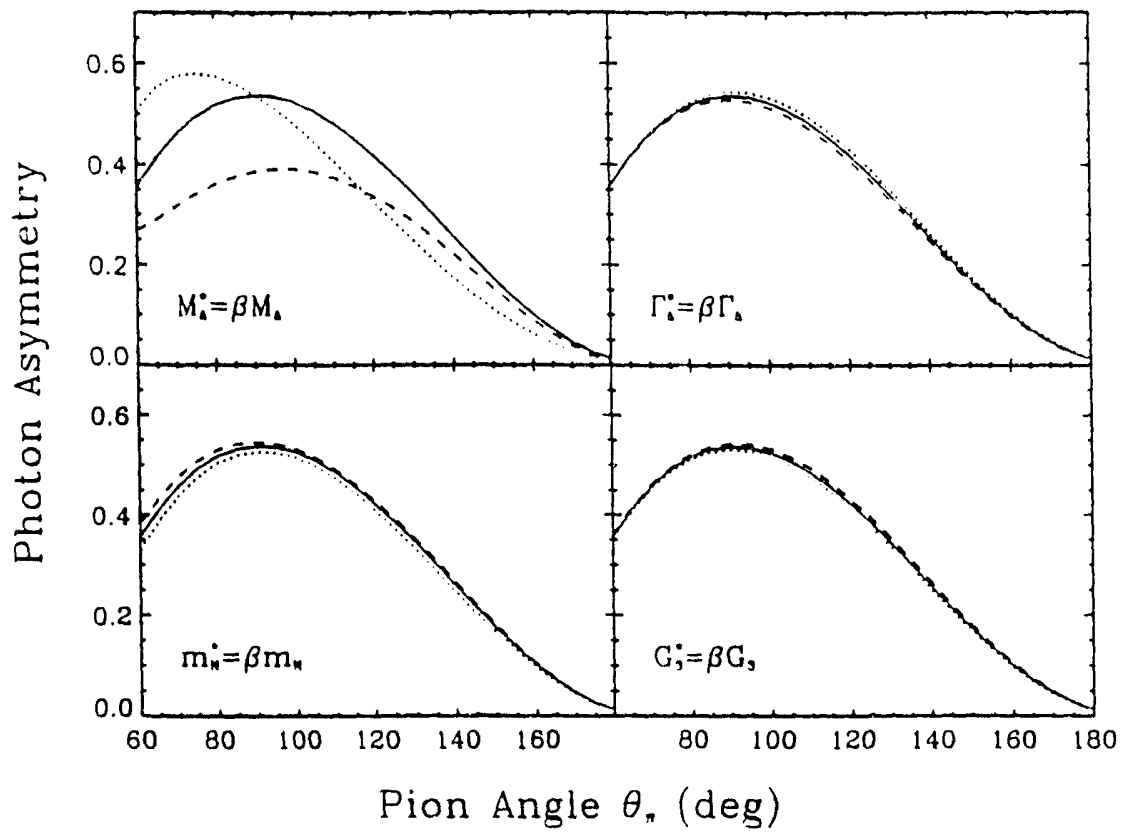

Fig. $\tau$ Same as Fig. 6, but for the photon asymmetry.

the curves. In the cases we have examined. nonlocal effects always enhance the local DWIA cross sections. However, the enhancements are not very significant. Including them improves the agreement between the calculations and the data for most cases.

We find a great sensitivity of the calculated cross sections and photon asymmetries to the $\Delta$ mass. Within our local DIVIA analysis. it appears to be able to explain the disagreement between the Bates data and theoretical predictions at the forward pion angle if the $\Delta$ mass is reduced by about $5 \%$. We must stress that this conclusion is rather preliminary. This ad hoc reduction in the $\lambda$ mass needs to be on firmer dynamical ground. and our result serves only to generate more interest in this problem.

We have successfully established a full nonlocal DWIA calculation for the reaction $A(\gamma, \pi N) B$ in the $\triangle$ region. The calculation is set up in a very general way so that each ingredient can be replaced by an alternative one if necessary. Comparisons with the existing data suggest that it contains the correct basic ingredients. More experiments are needed to fully investigate this very promising reaction and. in fact. such efforts are already in progress [8, 12].

Finally, we intend to extend the calculation to the virtual photon case. namely, pion electroproduction from complex nuclei $A\left(e . e^{\prime} \pi N\right) B$ in order to study the longitudinaltransverse response of the production process in the nuclear medium. Such an effo:t is underway. Beyond that. one can go to higher energies to study other meson production reactions. such as kaon production and eta production. One can also use our formalism to study the time reversal reartion. e.g., radiative pion capture by nuclei.

This work will appear in the dissertation of Xiaodong $\mathrm{Li}$. 


\section{References}

[1] X. Li and L. E. Wright, J. Phys. G 17, 1127 (1991).

[2] B. Mecking, CEBAF Proposal and Private Communication.

[3] C. Bennhold, Phys. Rev. C 38. 1944 (1989).

[4] Xiaodong Li, L. E. Wright, and C. Bennhold. Phys. Rev. C 45. 2011 (1992).

[5] Michael M. Tung, Multipole Decomposition of Kaon-Photoproduction Operators, M.S. Thesis, Ohio Lniversity, 1991.

[6] L. Tiator and L. E. Wright, Phys. Rev. C 30, 989 (1984); C. Bennhold. L.Tiator and L. E. Wright, Can. J. Pnys., 68, 1270 (1990).

[7] L. Tiator, J. Vesper, D. Lirechsel. N. Ohtsuka and L. E. Wright, Nucl. Phys. A485, 565 (1988).

[8] G. van der Steenhoven, in Proceedings of the 7th Annual NIKHEF Conference. Amsterdam, p.92, December (1991).

[9] I. V. Glavanakov and V. N. Stibunov, Sov. J. Nucl. Phys., 30(4), 465 (1979): I. V. Glavanakov. Sov. J. Nucl. Phys., 49(1), 58 (1989).

[10] I. V. Glavanakov. Sov. J. Nucl. Phys. 52(6), 205 (1990).

(11) L. D. Pham. S. Hoibrảten. R. P. Redwine. et al.. Phys. Rev. C 46. 621 (1992).

[12] Proposal to the LEGS facility. spokesmar. K. Hicks. 1992

[13] K. I. Blomqvist and J. ... Laget. Nucl. Phys. A280. 405 (197T).

[14] J. .. Laget, Nucl. Phys. A481. 765 (1987).

[15] R. Davidson. A. C. Mukhopadhyay and R. Wittman. Phys. Rev. Lett. 56. 804 (1986).

[16] K. Stricker. H. Míc.Manus, and J. A. Carr. Phys. Rev. C 19. 929 (1979); 22. 2043 (1980): 25. 952 (1982).

[17] P. Schwandt. H. O. Meyer. W. W. Hacobs, et al.. Phys. Rev. C 26. 55 (1982). 
(b) Coulomb distortion effects on $\left(e, e^{\prime} \gamma\right)$ and on inclusive $\left(e, e^{\prime}\right)$ and exclusive $\left(e, e^{\prime} p\right)$ scattering in the quasi-elastic region

We finished our work on the inclusion of Coulomb distortion effects in $\left(e, e^{\prime} \gamma\right)$ and the paper reporting these results has appeared [1]. We found excellent agreement with the data from an experiment performed at Mainz [2] on the $6.32 \mathrm{MeV}$ mixed E2-M1 transition in ${ }^{15} \mathrm{~N}$ and with an experiment from Illinois [3] on a $2^{+}$transition in ${ }^{12} \mathrm{C}$. While no data is yet available from medium and heavy nuclei, our analysis indicates that it will be possible to analyse mixed transitions even with substantial Coulomb distortion effects present. Note that our calculations include the bremsstrahlung contributions which is very large near the incoming or outgoing electron directions. This work formed part of the dissertation project of Amirul Hoque (Ph.D. 1992, Ohio University) who was supported by the Physics Department at Ohio University, and was done in collaboration with a former student. Indu Talwar who is now on the faculty at Geneseo (SUNY). The computer code for this investigation was written in a flexible manner and we anticipate extensive use of it when additional $\left(e, e^{\prime} \gamma\right)$ experiments are performed. This reaction has great potential for nuclear spectroscopy of low-lying nuclear states, particularly ones with mixed transitions to the ground state.

A calculation using Coulomb distorted waves for the electron has been described for the inclusive case, $\left(e, e^{\prime}\right)$ in a recent publication [4]. The inclusive case uses the same formalism as we used for the exclusive reaction $\left(e, e^{\prime} p\right)[5]$ except that we integrate over outgoing nucleon angles and sum over all single particle states (including neutrons). In addition. because we now include channels in which the nucleon is absorbed on the way out of the nucleus, we discard the imaginary part of the optical potential. Since electron plane wave calculations are normally done in the Coulomb gauge while we work in the Lorentz gauge, it is essential for comparison purposes to maintain current conservation and hence gauge invariance. This can achieved in this model by using the same real potential for the nucleons in the initial and final states. At the energies used here, there is little difference between this and the real part of the optical potential. More sophisticated treatments of the final state are possible in the plane-wave formalism [6] but. while concerned with the effects of Coulomb distortion. such niceties are not needed. For the bound state proton and neutron wavefunctions we use relativistic Hartree wavefunctions (see Section (c)).

For the case of ${ }^{40} \mathrm{Ca}$ we have shown [4] that the effective momentum approximation (E.MA) can be used in place of the full distorted-wave calculation. The form of the EMA we use (which is not standard) is given below.

$$
\left(\frac{d^{2} \sigma}{d \Omega d{ }^{2}}\right)_{E . M A}=\left(\frac{p_{e f f}^{i}}{p^{i}}\right)^{2}\left(\frac{d^{2} \sigma}{d \Omega d \omega}\left(p_{e f f}^{i}, p_{e f f}^{f}\right)\right)_{P W B A}
$$

where the effective momentum is defined by $p_{\text {eff }}^{i . f}=p^{i . f}+1.5 a Z / R$. 

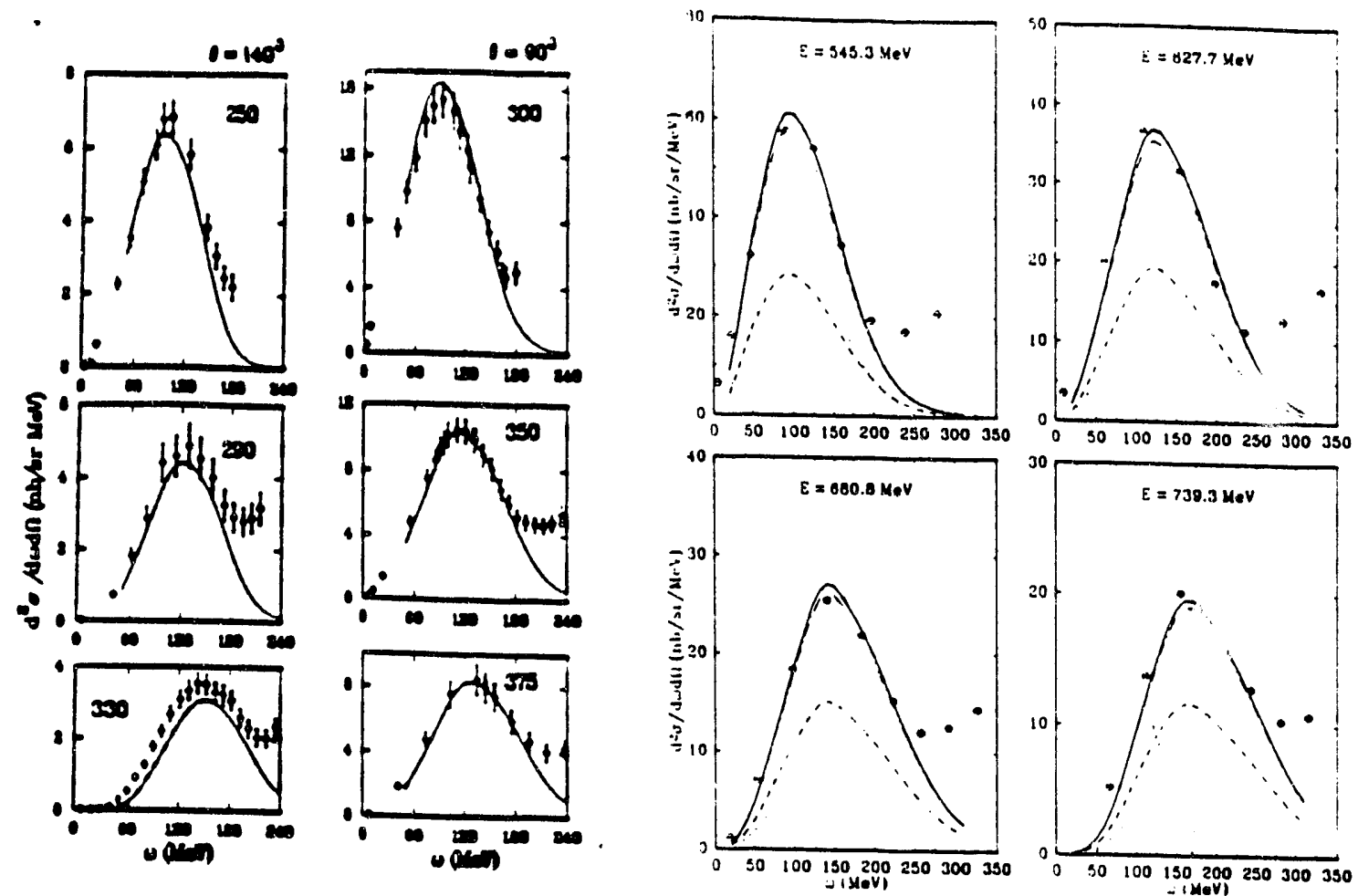

Fig. $1{ }^{40} \mathrm{Ca}$ data from Bates. Courtesy of $\mathrm{C}$. Blachley and $\mathrm{C}$. Williamson.

Fig. 2 Data from Ph.D. Dissertation of $T$. Yates. MIT, 1992. The solid line is the EMA result, the dotted line is the longitudinal contribution, and the dashed line is the transverse contribution. The long dash-dot curve is a best fit with a $12 \%$ suppression of the longitudinal contribution and a $3 \%$ enhancement of the transverse contribution.

We have shown [4] that the electron cross sections measured [ 7$]$ at a variety of energies and angles and plotted as a function of energy transfer. can be successfully compared with this calculation (Fig. 1). IVe have also remarked that these measurements are insensitive to the longitudinal structure function. Note that we also caution against extracting structure functions by the Rosenbluth procedure. Firstly, Coulomb corrections need to be made using the correct EMA approximation (even for ${ }^{40} \mathrm{Ca}$. errors of approximately $12 \%$ are made in the longitudinal structure functions $S_{L}$ if the plane wave Born approximation is used). Secondly, if the Rosenbluth procedure is used. it is important to have sufficient data so that most of the data points that contribute to the peak of the structure function come from near the peak of the cross section.

New measurements $[8]$ at forward angles $\left(\theta_{e}=45.5^{\circ}\right)$ which are inuch more sensitive to $S_{L}$ have now been made at a range of electron energies and energy transfers. We have compared all of this new data with our calculations and realized the importance of kinematically defining the quasi-elastic region in making the comparison. 

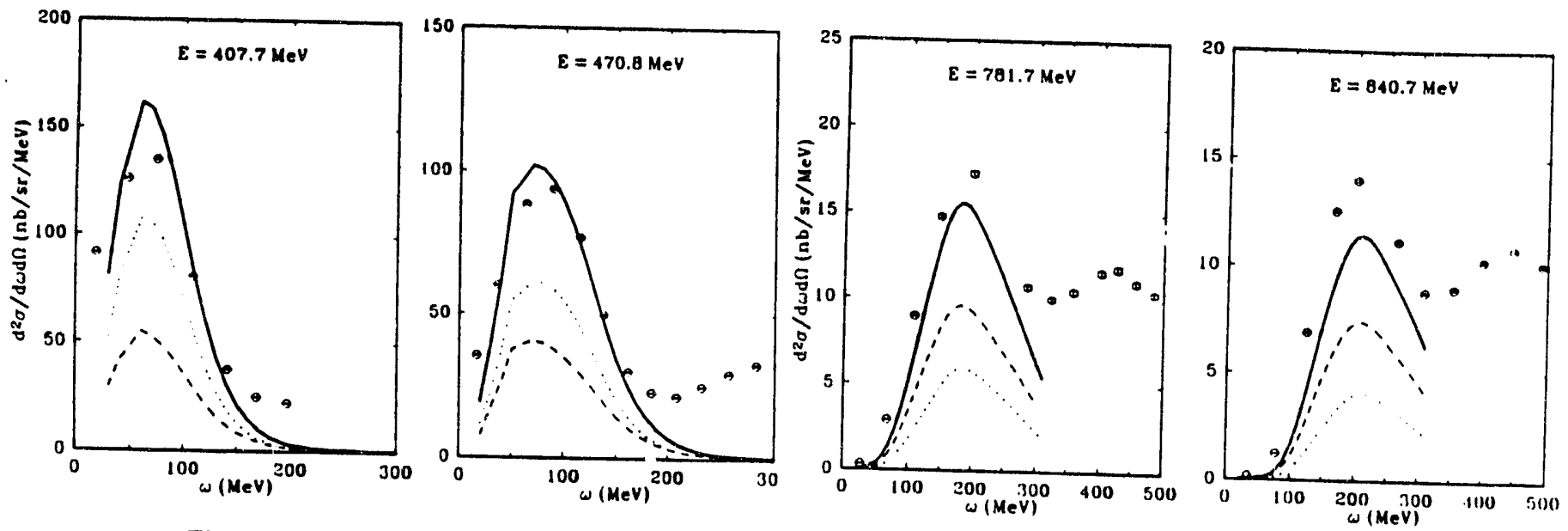

Fig. 3 Same as Fig. 2 except $\omega<100 \mathrm{MeV}$. Fig. 4 Same as Fig. 2 except $\omega>200 \mathrm{MeV}$.

Our present model only includes quasi-elastic nucleon knockout. It does not include any mechanism for exciting various giant resonances, nor for describing quasi-free pion production. Thus we should exclude from the comparison the lower energy transfer region, say $\omega<100 \mathrm{MeV}$ and the higher energy transfer region where pion production becomes important. say $\omega>200 \mathrm{MeV}$. In Fig. 2, we show the four energies measured at Bates with the peak between $100 \mathrm{MeV}$ and $200 \mathrm{MeV}$. We find quite good agreement between our single particle calculation and the data. We show our model predictions for the longitudinal and transverse contributions. and if we adjust a scale factor in front of each of these we obtain the best fit to the data shown in Fig. 2. We conclude that there is no evidence of appreciable longitudinal suppression in $\left(e, e^{\prime}\right)$ on ${ }^{40} \mathrm{Ca}$.

In Figs. 3 and 4 . we compare our calculations to the experimental data where the peak falls below $100 \mathrm{MeV}$ and above $200 \mathrm{MeV}$ respectively. Note that when $\omega$ is below $100 \mathrm{MeV}$. the calculation exceeds the data while when $\omega$ is above $200 \mathrm{MeV}$ the calculation falls below the data as expected since pion production is an incoherent process which has not been included.

In the case of ${ }^{238} U$. Coulomb distortion is too strong for the E.MA to work. This is evident in Fig. $J$ where we compare plane-wave distorted-wave and E.MA calculations (Note: in $p_{\text {eff }}$ the factor 1.5 has been changed to 1.0 to improve the E.MA). An account of the experiment on ${ }^{238} \mathrm{C}$ and the Rosenbluth-extracted structure functions are given in Ref. [6]. however the cross section data shown here are not published [9]. In Fig. 6 


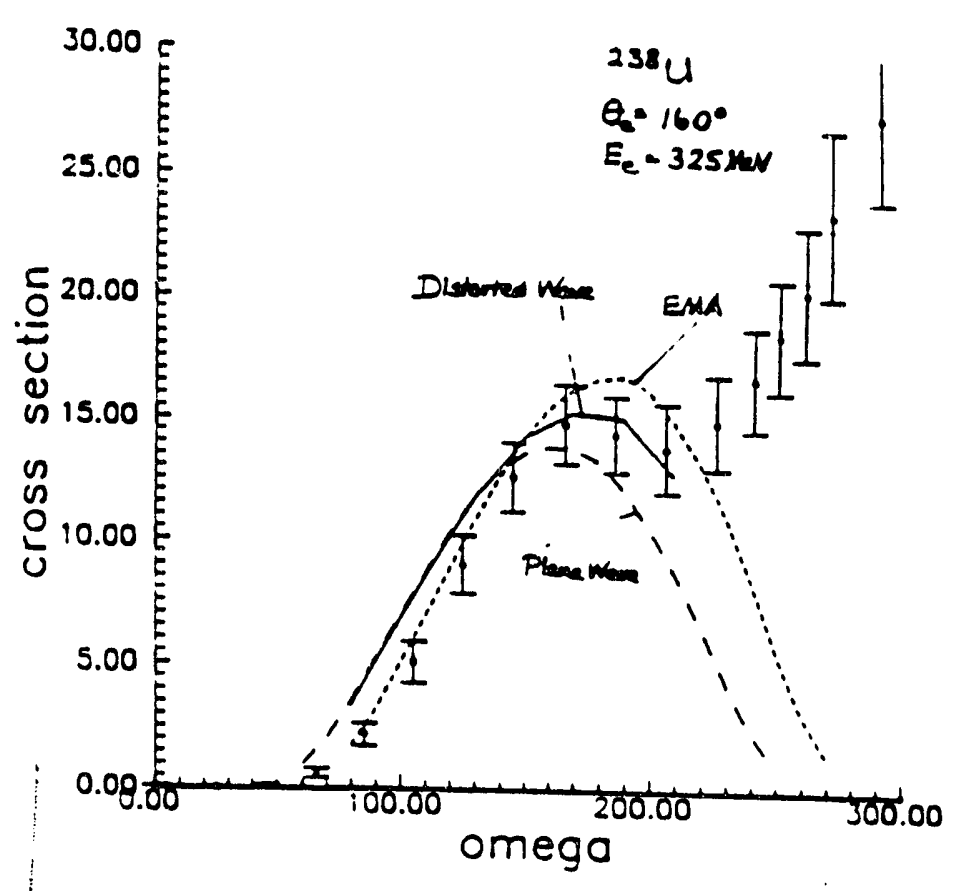

Fig. 5 Solid line is DWBA, Dashed line is EMA and dotted line PIVBA.

we compare our distorted wave calculations with the data. Although the agreement is poor, the only case of hopeless disagreement is data for $\theta=90^{\circ}$ and these, we are advised, are highly suspect [10]. There is no evidence of longitudinal suppression. which was also the conclusion of Ref. [6].

A fully distorted partial wave calculation has been carried out [5] for the quasielastic $\left(e . e^{\prime} p\right)$ reaction which uses a single particle wavefunction from a relativistic Hartree model [12] to describe the initial bound proton. and a global optical potential [15] to describe the final-state interaction of the knocked-out proton with the residual nucleus. Coulomb distortion of the electrons is also included. The cross section calculation which treats all other nucleons as spectators is called the single-particle transition cross section. To compare with the experimental data. we define [5] the reduced cross section in terms of the DWBA single-particle cross section as

$$
\rho_{m}\left(\vec{p}_{m}\right)=\frac{S_{\kappa_{b}}}{p E \sigma_{e p}} \frac{d^{5} \sigma}{d k_{0}^{\prime} d \Omega_{k^{\prime}} d \Omega_{p}} .
$$

In Eq. (2) $\sigma_{e p}$ is the electro-proton cross section [16] and $S_{\kappa_{b}}$ is the spectroscopic factor. One can use $S_{\kappa_{b}}$ as a scaling factor to fit to the experimental data. Over the past few years. spectroscopic factors for several nuclei have been extracted [1T] and are commonly low compared with those extracted using other probes. All data shown here is from experiments at NIKHEF [13, 18].

We want to know whether. by treating the electron Coulomb distortion correctly, 


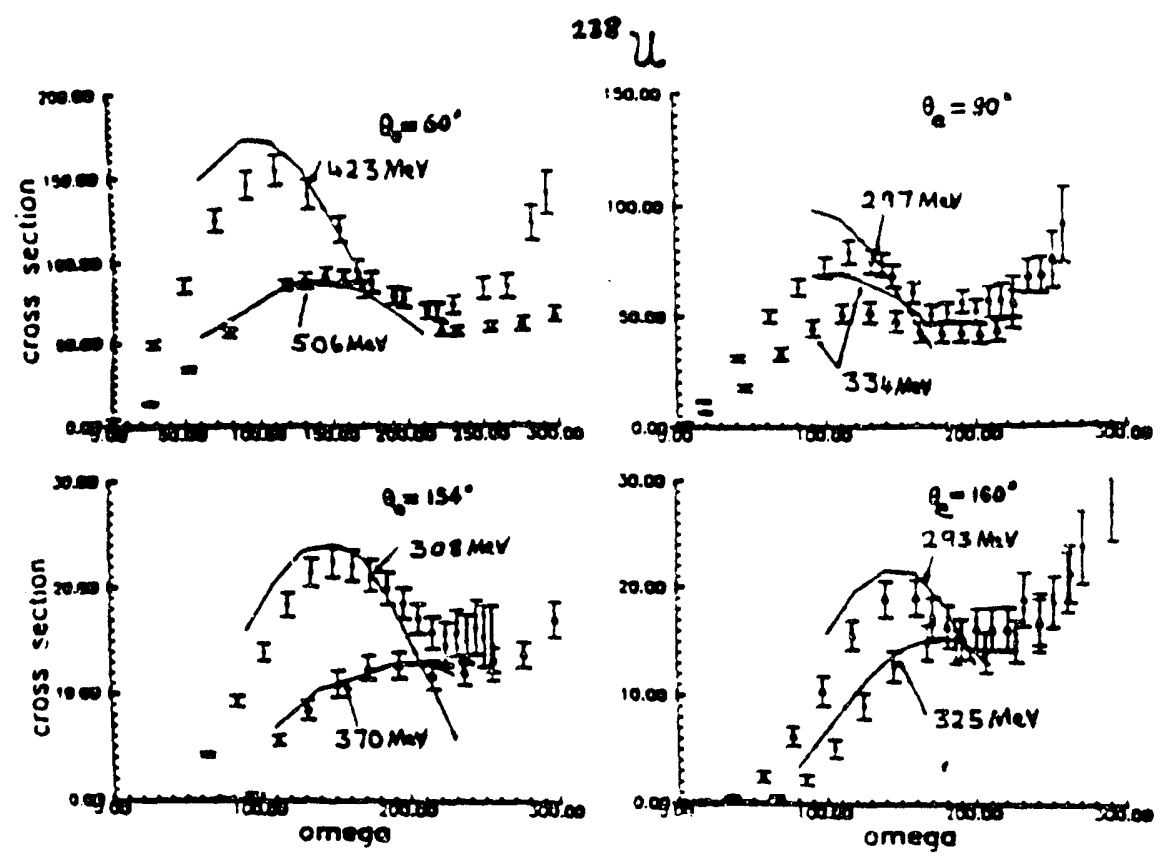

Fig. $6{ }^{238} U$ data, Courtesy of C. Blachley and C. Williamson.

our calculation can provide spectroscopic factors different from the previous calculations. In Fig. 7 , we show a calculation for knocking a proton from the $3 s_{1 / 2}$ state of ${ }^{208} \mathrm{~Pb}$. The solid curve is our DWBA calculation. the dotted line is the plane wave calculation. and the dashed line is the DWBA calculation with $Z=1$. These clearly show that the electron Coulomb distortion is essential to shift the maximum and minimum to the right place to describe the data. All these curves are scaled by $S_{3 s_{1 / 2}}=0.71$ which is obtained by a least-squares fit. The earlier value [13] was 0.51 .

Spectroscopic factors obtained from fitting to our calculation are usually larger than the ones previously obtained $[5,14]$ from the same seaction. This is partially due to the electron Coulomb effects and partially due to the relativistic formalism.

To calculate these spectroscopic factors directly, we rewrite the cross section using many particle wavefunctions. We can define an effective single particle initial state (take ${ }^{40} \mathrm{Ca}$ as an example).

$$
\Phi_{i}=<\left.{ }^{39} \mathrm{Ki}^{-*}\right|^{40} \mathrm{Ca}>
$$

where we use " to indicate the final state of the residual nucleus. The effective single particle state is neither a ${ }^{39} \mathrm{~K}$ nor a ${ }^{40} \mathrm{Ca}$ single particle state. and to calculate this. we have to know the entire wavefunctions for both the initial target nucleus and the final $A-1$ nucleus.

We used our odd-A Hartree relativistic code to calculate the ground state wavefunction of the ${ }^{39} K$ [12] which expands the wavefunction in terms of ${ }^{40} \mathrm{Ca}$ singleparticle wavefunctions. For knocking out a proton from $d_{3 / 2}$ state of the ${ }^{40} \mathrm{Ca}$ target 


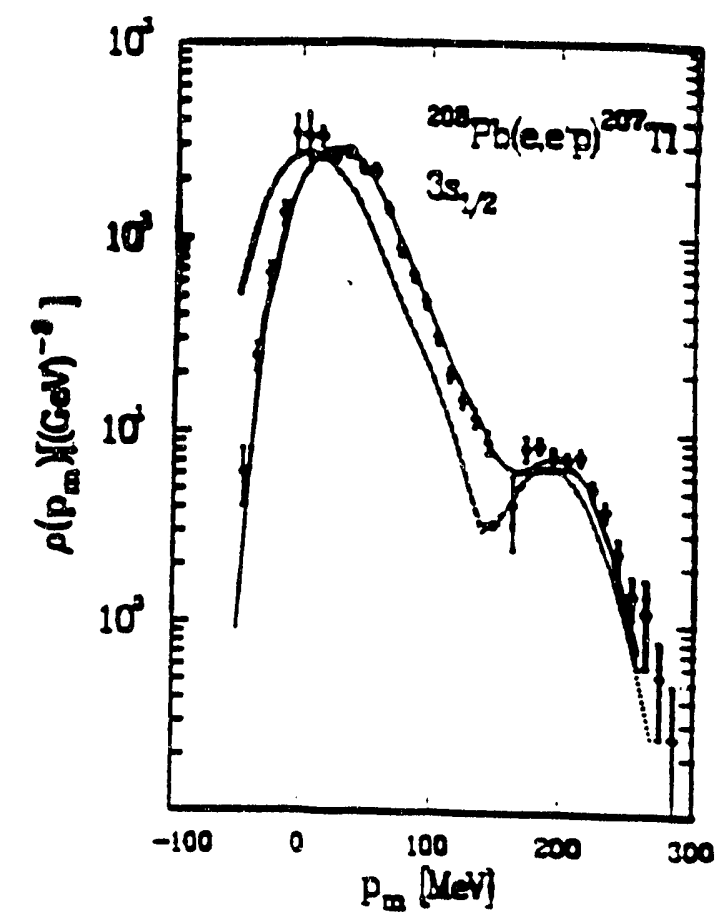

Fig. $T$ Parallel kinematics calculation for ${ }^{208} \mathrm{~Pb}$, data from Ref. (18).

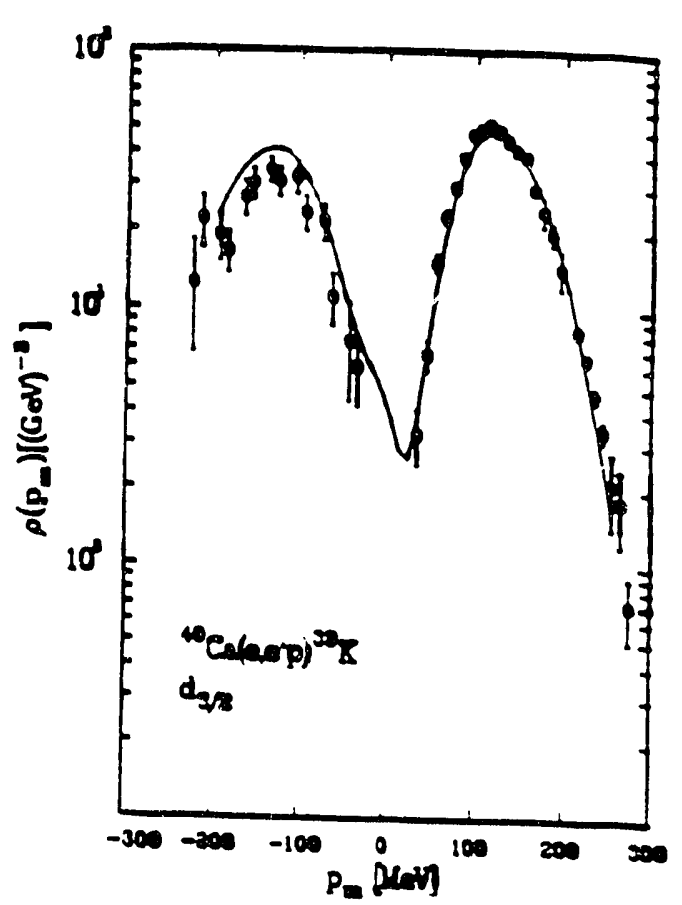

Fig. 8 Parallel kinematics calculation for ${ }^{40} \mathrm{Ca}$, data from Ref. [13]. leaving ${ }^{39} \mathrm{~K}$ in its ground state, the effective single particle wavefunction can be writ-
ten

$$
\begin{aligned}
& \Phi_{i}=\left\langle\left.{ }^{39} \mathrm{~K}\right|^{40} \mathrm{Ca}\left(d_{3 / 2}\right)^{-1}>\psi_{d_{3 / 2}}+<\left.{ }^{39} \mathrm{~K}\right|^{40} \mathrm{Ca}\left(2 s_{1 / 2}\right)^{-1}>u_{2 s_{1 / 2}}+\cdots\right.
\end{aligned}
$$

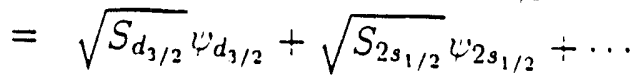

$S_{d_{3 / 2}}$, defined above, is the spectroscopic factor. For this particular reaction. it turns out that only the first term is needed and the spectroscopic factor is defined by

$$
S_{d_{3 / 2}}=<\left.{ }^{39} K\right|^{40} \mathrm{Ca}\left(d_{3 / 2}\right)^{-1}>\text {. }
$$

The parameters needed for the Hartree calculation and the optical model are determined by other considerations and hence. from our viewpoint, the reduced cross section calculated in this way, is a parameter-free description of the $\left(e, e^{\prime} p\right)$ process. In Fig. 8 we show the calculated cross section compared with the experimental data [18]. The calculated spectroscopic factor is 0.81 which happens to agree exactly with the one we would obtain by just scaling our single-particle result to fit the data. Since in our model the states below the Fermi sea are completely filled, this result suggests that there is no appreciable depletion of the $d_{3 / 2}$ state.

We also looked at the proton knocked-out from the $2 s_{1 / 2}$ state in ${ }^{40} \mathrm{C} a$ which leaves the residual nucleus in an excited state. The calculated spectroscopic factor is again 


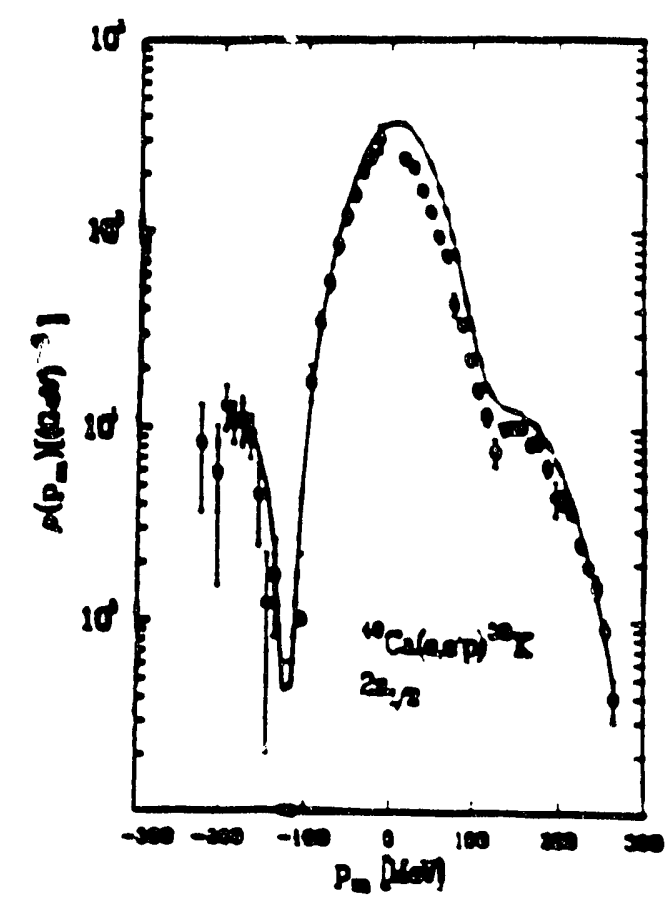

Fig. 9 Same as Fig. 8 but for $2 s_{1 / 2}$ state.

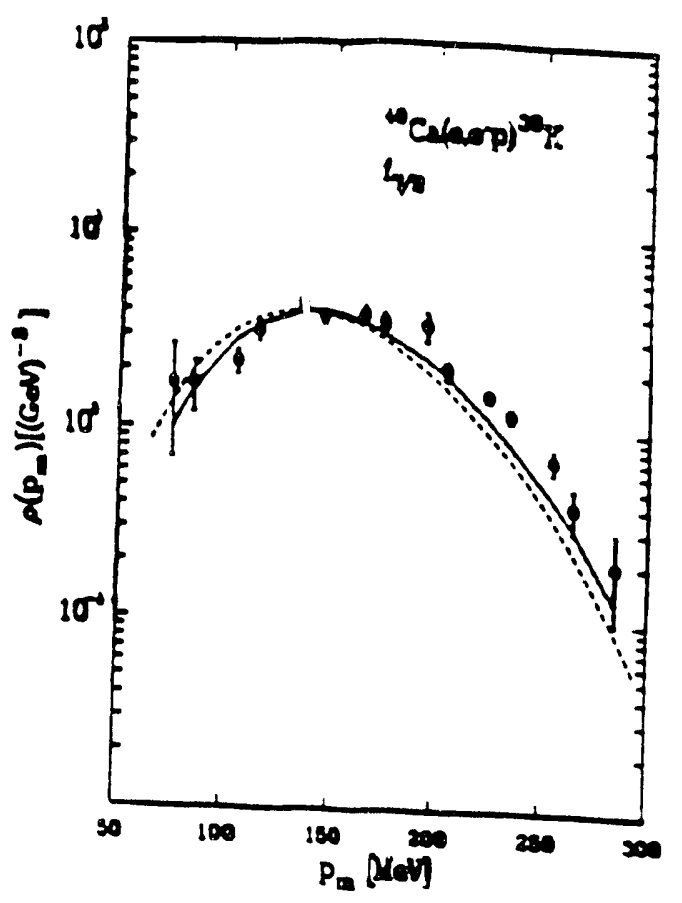

Fig. 10 Same as Fig. 8 but for $f_{7 / 2}$ state.

0.81 and in Fig. 9 we show the calculation and the data. The agreement is not nearly as good as for the $d_{3 / 2}$ case. The shape from our model calculation around the main peak is noticeably different from the experimentally observed one. If one used our single particle wavefunction calculation to fit the data. the extracted spectroscopic facte: vould be 0.55 . but the shape discrepancy would still be there.

Experimentally there appears to be a contribution from the $f_{i / 2}$ state. which is the first state above the Fermi sea. In Fig. 10 we show our single particle calculation compared with the data. The extracted $S_{f_{i / 2}}=0.037$ which is smaller than the value quoted by Kramer [13]. In our nuclear model the factor would be zero and thus a model of the spectroscopic factor for this state requires an RP.i or equivalent calculation bevond the Hartree or Hartree-Fock which we have not yet done.

\section{References}

[1] Indu Talwar. Amirul Hoque. L. E. Wright. Phys. Rev. C44, 2694 (1991); op.cit. C46. 822 (1992).

[2] Rainer Nick, diploma. University of Mainz, 1988.

[3] C. ․ Papanicolas et al.. Phys. Rev. Lett. 54. 26 (1985).

[4] Y. Jin. D.S. Onley and L.E. Wright. Phy. Rev. C45, 1333(1092). 
[5] Y. Jin. D.S. Onley and L.E. Wright. Phy. Rev. C45. 1311(1992).

[6] P.M. Boucher and J.E. Van Orden, Phy. Rev. C43, 582(1991).

[7] .I. Deady et al., Phy. Rev. C33, 1897(1986); Phy. Rev. C28, 631(1983).

[8] T.C. Yates, Ph.D. Dissertation. MIT, 1992.

[9] C.C. Blatchley, J.J. LeRose, O.E. Pruet. P.D. Zimmerman, C.F. Williamson, and M. Deady, Phy. Rev. C34. 1243(1986).

[10] C.C. Blatchley and C.F. Williamson, private communication.

[11] S. Boffi, C. Giusti, F.d. Pacati and S. Frullani, Nucl. Py. A319, 461(1979); S. Boffi, C. Giusti and F.d. Pacati, Nucl. Phy. A336, 437(1980); C. Giusti and F.d. Pacati, Nucl. Phy. A336, 427(1980)

[12] J.K. Zhang and D.S. Onley, Phys. Rev. C43, 942(1991).

[13] G.J. Kramer, Ph. D. dissertation, University of Amsterdam, 1990.

[14] Yanhe Jin, Ph. D. dissertation, Ohio University 1991.

[15] S, Hama, B.C. Clark, E.D. Cooper, H.S. Sherif and R.L. Mercer. Phy. Rev. C41. $2737(1990)$

[16] T.D. DeForest, Jr., Nucl. Phy. A392, 232(1983)

[17] S. Frullani and J. Mougey, it Adv. in Nucl. Phy., vol, 14, edited by J.W. Negele and E.Vogt (Plenum. New York, 1984).

[18] Louk Lapikás. private communication: Edwin Quint. Ph. D. dissertation. University of Amsterdam. 1988.

\section{(c) Relativistic Structure Calculations}

In these calculations the basic lagrangian is an extension of the $\sigma-\omega$ model in which we couple the nucleons to five fields. $\sigma . \omega, \rho, \pi$ and the electromagnetic field. Tensor coupling to the vector mesons, $\rho$ and $\omega$, is also considered. The unique feature of our approach is that we first solve the Hartree problem with the nucleus constrained to be spherical. which can be done easily in the non-spectral approach. and then use the states so obtained as the basis for a spectral expansion. At this point we may admit nuclear deformation. polarization and exchange terms as appropriate. In the event an even-even nucleus has a truly spherical ground state the Hartree calculation will simply show that basis states are the solutions. For nuclei which are slightly 
deformed or differ from a spherical nucleus by a single-particle or single-hole we find the number of basis states required in this formalism is relatively small which is a great advantage. Also in treating nuclear reactions involving neighboring nuclei we have the advantage of having nuclear states expressed in the same basis: we have used this to good effect in $\left(e, e^{\prime} p\right)$ reactions.

Hartree Calculations-Early in the grant period we finished a systematic study of deformed nuclei in the s-d shell $[1,2]$; both even-even and odd-A nuclei were included. For even-even nuclei we made comparisons with the quadrupole moments and binding energies. For odd-A nuclei the core is spontaneously polarized by the space-like components of the vector fields and there is an additional contribution from the pion. These results showed that the pion contribution had fairly large effects on magnetic moments especially for the hole nuclei, which appeared however to be in the opposite direction to that, for meson exchange corrections [3]. The latter can only be calculated in a manner compatible with the full self-consistent mean-field calculation at the level of a Hartree-Fock calculation, where the exchange terms admit a contribution from charged mesons.

Generally we found that tersor couplings for the vector mesons play a negligible role at the Hartree level $[1,2]$.

We have since developed a Hartrec code which allows for non-axial deformation of the nucleus. These calculations have turned up no seriously deformed non-axial ground states in the $s-d$ shell. We have found small non-axial effects in ${ }^{24} M g$ at the level where they are imperceptible but reproducible in our calculation. We have done some pilot calculations in the $f-p$ shell where non-axial shapes reportedly occur [4]. We feel we need further understanding of the role of pairing terms. which are usually introduced at this level but are not natura!!y present in the mean-field hamiltonian.

Hartree-Fock Calculations-Many of our results found in the course of Hartree calculations strongly suggested that we would be more confident of our results if we were to include the Fock terms. Our codes have the capacity to include the exchange terms in almost all the calculations described above, but the increase in the memory size and running time have confined us to dealing with the simplest cases. Thus we hive completed a Hartree-Fock study of even-even deformed nuclei in the s-d shell [5]. We are restricted in our conclusions by the absence of a well-chosen set of coupling parameters for the relativistic Hartree-Fock case. We have used a set of parameters fixed by consideration of the saturation of nuclear matter [6] but which are not optimal for finite nuclei, and we will need to est.ublish a set of coupling constants and, where appropriate, effective meson masses, by fitting to spherical nuclei throughout the periodic table.

We have looked at some features unique to the Fock terms. The first of these is retardation of the meson propagators [i] which, we find, has an appreciable effect only in the contribution of the pion (being the lowest mass meson), but increases with nuclear mass (because the average energy difference between any exchanging pair of states increases). The second feature is the meson exchange current and the enhanced 
role of the isovector mesons in odd-A nuclei. We have calculated the wavefunctions for odd-A nuclei in the $s-d$ shell which differ from a closed shell by one-hole or one particle $\left({ }^{15} N,{ }^{17} O,{ }^{17} F,{ }^{39} \mathrm{~K}\right.$, etc. $)$ and are in the process of including the meson currents in the magnetic moment calculation. We have also reexamined the tensor con-ling of the. $\rho$-meson and find, in this regard, some serious difference with other work [8] which we have been unable to resolve.

We also published [9] a short study of an interesting alternative (derivative) type coupling of the nucleon and the $\sigma$ field, originally proposed for bulk nuclear matter. Here we found results for finite nuclei were unsatisfactory because the spin-orbit coupling was too small.

Most recently we completed the first temperature-dependent relativistic HartreeFock calculation [10]. We use the same basic lagrangian and determine the occupation numbers of the states by minimizing the grand potential at fixed temperature. We looked at the temperature dependence of excitation energy, single particle spectra, charge distribution, and entropy, for two spherical nuclei, ${ }^{16} \mathrm{O}$ and ${ }^{40} \mathrm{Ca}$. In general we found thermal response somewhat larger than nonrelativistic results.

\section{References}

[1] Jian-Kang Zhang and D.S. Onley, Phys. Lett. 209B 145 (1988).

[2] Jian-Kang Zhang and D.S. Onley, Nucl. Phys. A526, 245(1991).

[3] T.M. Morse, C.E. Price and J.R. Shepard Phys. Lett. 251B, 241 (1990).

[4] J.A. Sheikh et al.. Phys. Rev. Lett 64. 376 (1990); C.J. Lister et al., Phys. Rev. C 42 R1191, (1990).

[5] Jian-Kang Zhang and D.S. Onley. Phys. Rev. C 43, R942 (1991).

[6] P. G. Blunden and M.J. Iqbal. Phys. Lett. B 196. 295 (1987).

[7] Jian-Kang Zhang and D.S. Onley. Phys. Rev. C 44, (1991) 1915.

[8] P. G. Blunden. Proceedings of the Workshop at the Ohio State Lniversity Relativistic Nuclear Many-body Physics. p 265.

[9] Jian-Kang Zhang and D.S. Onley. Phys. Rev. C 44, 2230(1991).

[10] Jian-Kang Zhang and D.S. Onley, Phys. Rev C 46. 167T (1992). 


\section{(d) Quark Models}

The kaon photoproduction process has been studied using a phenomenological photoproduction operator [1] as a potential probe of nuclear structure. We investigated [2] the interaction

$$
\gamma+p \rightarrow K^{+}+\Lambda^{0}
$$

in a simple quark model which supplies the coupling constants and form factors associated with each vertex in the Feynman diagrams and compared with the phenomenological model. The model is an extension of the nonrelativistic Isgur-Karl model [3] and is similar to the Quark-Pair-Creation (QPC) model of Le Yaouanc et al. [4] but does not have the arbitrary coupling constants present in that model. We set up a nonrelativistic quark model Hamiltonian which has the general form:

$$
\begin{aligned}
H= & \sum_{i}\left[\frac{1}{2 m_{i}}\left(\mathbf{p}_{\mathbf{i}}-q_{i} \mathbf{A}_{i}\right)^{2}+m_{i}+\mu_{i} \sigma_{i} \cdot \nabla \times \mathbf{A}_{i}\right]+\sum_{i j} V_{i j}^{(2)} P^{(2)}+\sum_{i j k} V_{i j k}^{(3)} P^{(3)} \\
& +\sum_{i}\left[\tau_{i}^{(\text {prod })} \cdot\left(\mathbf{p}_{\mathbf{i}}-q_{i} \mathbf{A}_{i}\right)+\text { h.c. }\right]
\end{aligned}
$$

This is a semirelativistic model in that we allow particle production and annihilation in the electromagnetic coupling obtained by reduction of a Dirac Hamiltonian while describing quarks as nonrelativistic spin $\frac{1}{2}$ particles. The Hamiltonian is Hermitian and gauge invariant. The projection operators $P^{(2)}$ and $P^{(3)}$ select out the 2 and 3 body color singlets and $V_{i j}$ and $V_{i j k}$ are the 2 and 3 body harmonic oscillator potentials. The particle wavefunctions are obtained by putting together the appropriate quark spatial, flavor, spin, color wavefunctions. We assume that the baryons are composed of 3 quarks and mesons of a quark antiquark pair. The additional terms involving $q \bar{q}$ pair admixtures are omitted.

In the process (1), the $s \bar{s}$ quark pair may be created directly by the photon or the photon may be absorbed by any of the quarks accompanied by the creation of the $s \bar{s}$ pair in the walls of the confining potential (Figure 1(A)). Our quark model will also describe excited hadronic states, thus when the photon is absorbed by any of the 3 quarks which make up the proton, we can include intermediate $N^{*}$ states. Likewise if absorbed by any of the quarks in the $K^{+}$or $\Lambda^{0}$, the $K^{-*}$ and the $\Sigma^{0}$ or $Y^{-*}$ can appear as intermediate states. Corresponding to the quark flow diagrams, we can draw the conventional Feynman diagrams (Figure 1(B)) and calculate the coupling constants at the vertices.

The amplitudes corresponding to different initial and final spin states are calculated in the center of momentum of the $i p$ system. In the simplest form of the model we take the masses of the $u, d$ and $s$ quarks to be the same and include only the Born terms and the $\mathrm{K}^{*+}$ and $\Sigma^{0}$ intermediate states. The cross section is then calculated. Figure $2(A)$ shows a plot of the calculated differential cross section as a function of 


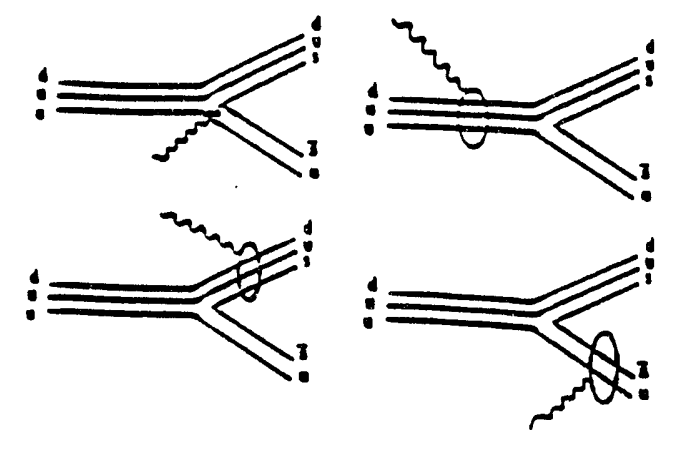

(a)

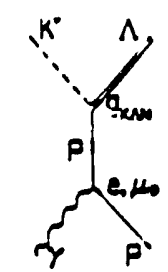

(c)

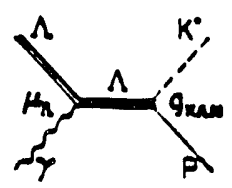

(4)

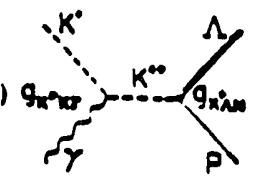

(b)

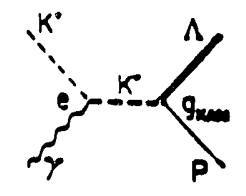

(d)

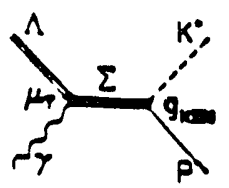

(i)

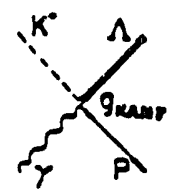

Fig. 1 (A) Quark flow diagrams for the kaon photoproduction process showing the photon absorption by any of the quarks. (B) Feynman diagrams. (a)-(c) represent the Born terms. (d) and (e) are the
$\Sigma^{0}$ and $K^{*+}$ exchange terms. (f) is the direct term.

the center of momentum angle $\theta_{c m s}$ at the photon energy of $1.2 \mathrm{GeV}$ in the laboratory as compared with the experimental data [5]. Differentiating the $s$ quark mass turns out to be significant as can be seen in Figure 2(B). This cross section also includes the contribution of the the Roper resonance $\mathrm{N}(1440)$.

The amplitudes of the various other $N^{*}$ and $Y^{*}$ intermediate states can be similarly calculated and are being included in our current calculation. Looking at the contribution from each of the various terms, we see that the Born terms account for most of the contribution, the $\Sigma^{0}$ and $K^{+*}$ for much of the remaining. Although, the $\mathrm{N}(1650)$ is above threshold, if the strict SU(3) classification of this resonance as ${ }^{4} 8$ were used there would be no contribution due to the Moorhouse selection rule [6]. Yet the mixing of the assignments of the $N(1650)$ and the $N(1535)$, which are both $\frac{1}{2}^{-}$ states, will create a contribution recognizable as a resonant peak and directly related
to the mixing angle.

Looking at the $\Lambda$ polarization, in a strict second-order perturbative calculation, polarization comes from orbital promotion of a quark (i.e. from certain resonant intermediate states). This observation is usually masked by the practice of putting in a complex energy (corresponding to the measured mass and width) into the propagator of any unstable particle. This gives an imaginary part to an amplitude which might otherwise be real. which in turn leads to a prediction of polarization from quite another cause. Including intermediate states where one or more quarks are excited from the ground state. gives a complex amplitude. However the contribution from such terms seem to be small and we anticipate that they will not make a significant contribution to the differential cross section for the process. This contrasts with the considerable contribution required by the phenomenological model [1].

We have some latitude in the choice of quark masses and hadron sizes but we have 

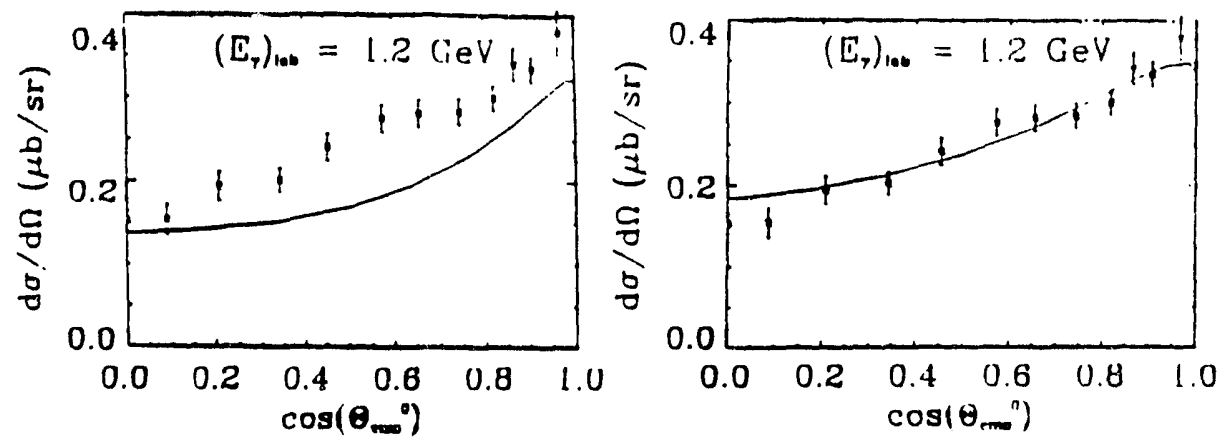

Fig. 2 Comparison of differential cross section in c.m.s. with experimental data : (A) taking the masses of the $u, d, s$ quarks the same and including the Born terms and the $K^{*}+$ and $\Sigma^{0}$ intermediate states. (B) taking the proper $s$ quark mass and also including the Roper resonance.

not yet used these to optimize our model.

\section{References}

(1) R. A. Adelseck. C. Bennhold and L. E. Wright, Phys. Rev. C 32, 1681 (1985).

[2] A. Kumar and D. Onley, Bull. Am. Phys. Soc. 37. 1319 (1992).

[3] N. Isgur and G. Karł, Phys. Rev. D 18. 4187 (1978).

[4] A. Le Yaouanc. L. Oliver, O. Pène and J.-C. Raynal. Phys. Rev. D 8. 2223 (1973).

[5] C. IV. Peck. Phys. Rev. 135. B830 (1964).

[6] R. G. Moorhouse. Phys. Rev. Lett. 16. T72 (1966). 


\section{Graduate Degrees Awarded During the Grant Period}

\section{Dissertations}

- Yanhe Jin, Ph.D. Electron Distortion Effects in Quasi-elastic Electron Scattering, Ohio University, March, 1991.

- Jian-kian Zhang, Ph.D. Relativistic Hartree-Fock Calculations of Deformed Nuclei in the s-d Shell, Ohio University, August 1991.

- Amirul Hoque, Ph.D. Coulomb Distortion Effects in $\left(e, e^{\prime} \gamma\right)$ Processes, Ohio University, December 1991.

- Xiaodong Li and Anita Kumar are expected to complete their Ph.D.'s by August of 1993.

\section{Master Theses}

- Michael M. Tung, Multipole Decomposition of Kaon-Photoproduction, Ohio University, December, 1990. 

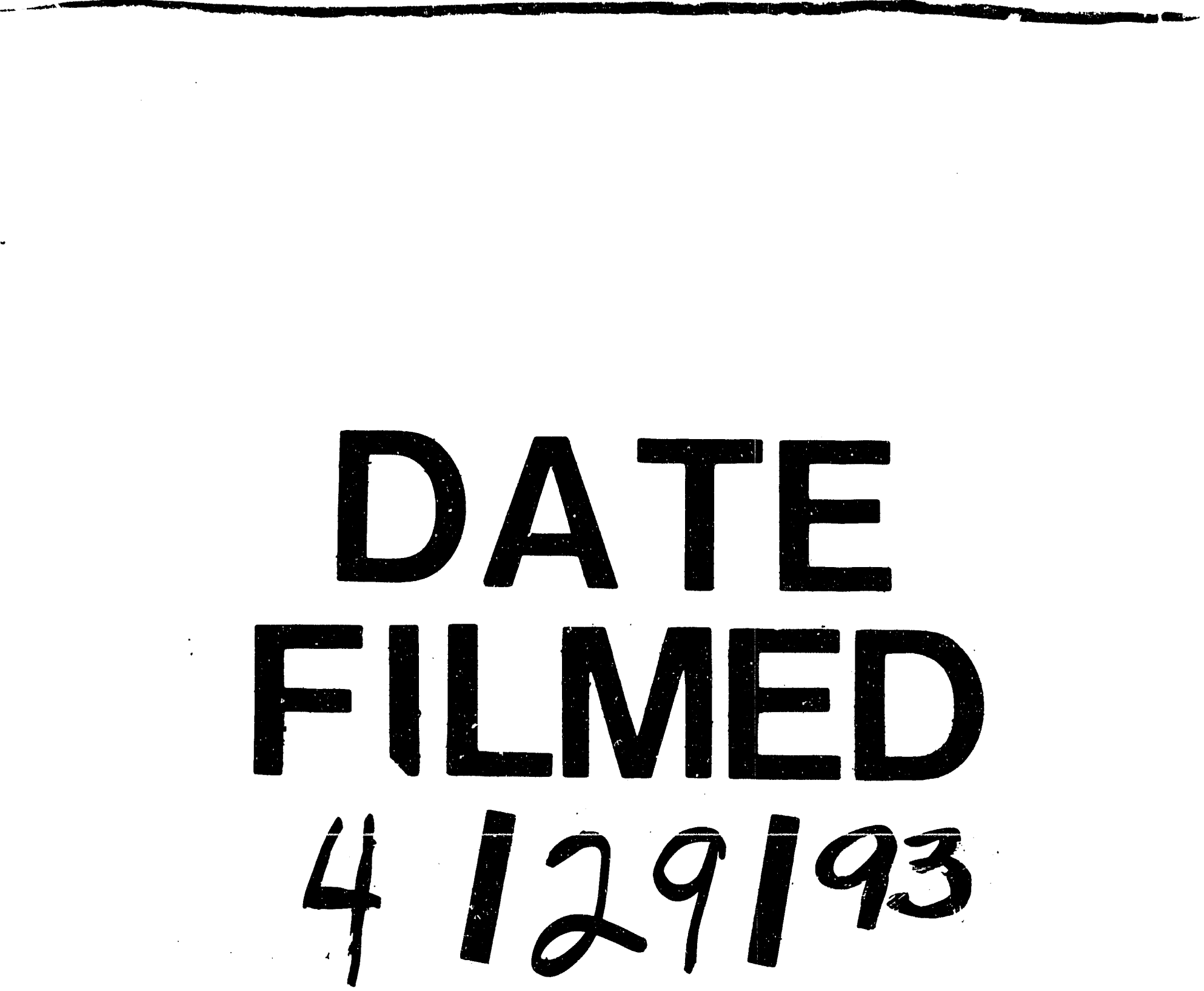
